\title{
Eficacia de las combinaciones antimicrobianas para el tratamiento de infecciones enterocócicas: una revisión crítica
}

\author{
Ivone Jiménez Toro', Carlos A. Rodríguez y y Andrés F. Zuluaga
}

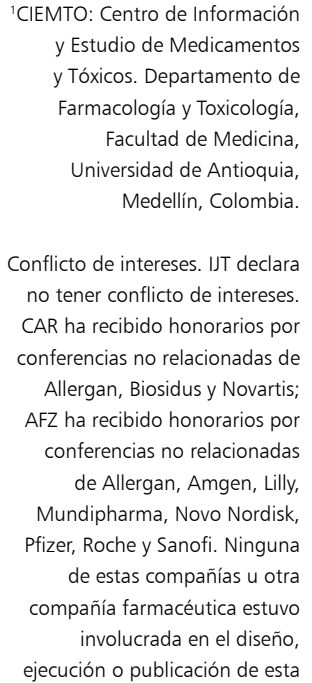

'CIEMTO: Centro de Información y Estudio de Medicamentos y Tóxicos. Departamento de Farmacología y Toxicología, Facultad de Medicina, Universidad de Antioquia, Medellín, Colombia.

Conflicto de intereses. IJT declara no tener conflicto de intereses. CAR ha recibido honorarios por conferencias no relacionadas de

Allergan, Biosidus y Novartis;

AFZ ha recibido honorarios por conferencias no relacionadas de Allergan, Amgen, Lilly, Mundipharma, Novo Nordisk, Pfizer, Roche y Sanofi. Ninguna de estas compañías u otra compañía farmacéutica estuvo involucrada en el diseño, ejecución o publicación de esta

revisión.

Fuente de financiación: Departamento Administrativo de Ciencia, Tecnología e Innovación COLCIENCIAS (contrato 111571149738) y convocatoria 785 (Doctorados nacionales COLCIENCIAS).

Recibido: 8 de agosto de 2018 Aceptado: 21 de Julio de 2019

Correspondencia a: Andrés F. Zuluaga Salazar andres.zuluaga@udea.edu.co

\section{Introducción}

$\mathrm{E}$ n las últimas tres décadas, especies del género Enterococcus han emergido como importantes agentes etiológicos de infecciones nosocomiales (Centers for Disease Control and Prevention, 2013). En los Estados Unidos de América se le atribuyen a estos patógenos más de 66.000 infecciones por año, de las cuales 22.000 son causadas por Enterococcus resistente a vancomicina (VRE, por su sigla en inglés) y más de 1.300 muertes están asociadas con cepas fármaco-resistentes ${ }^{1}$.

Enterococcus faecalis y Enterococcus faecium, son las dos especies de mayor relevancia clínica: el primero representa aproximadamente $80 \%$ de los aislados enterocócicos que afectan principalmente a pacientes críticamente enfermos, inmunocomprometidos o de edad avanzada $^{2}$. Estos patógenos causan una amplia variedad de infecciones que incluyen: bacteriemias, endocarditis, osteomielitis, infecciones de la cavidad abdominal, pélvicas, de tejidos blandos e infecciones urinarias ${ }^{3,4}$. Las bacteriemias y la endocarditis infecciosa (EI) son las infecciones más graves y difíciles de tratar, con tasas de mortalidad entre 28 y $75 \%$ en bacteriemia ${ }^{5}$ y en EI entre 20 y $40 \%$.

Diferentes factores de virulencia contribuyen a la patogénesis de las infecciones enterocócicas. La adhesión, colonización e invasión de los tejidos del hospedero y la producción de toxinas aumentan la gravedad de la infección. Enterococcus faecalis es la especie más virulenta y forma biopelículas con mayor frecuencia en comparación con E. faecium (87-95\% vs 16-29\%, respectivamente) $)^{7,8}$. La expresión de otros rasgos de virulencia, incluidos la proteína de superficie de Enterococcus (Esp), sustancia de agregación, citolisina y gelatinasa, también son más frecuentes en E. faecalis y contribuyen a que esta especie presente tasas más altas de morbilidad y mortalidad 9 .

Enterococcus puede adquirir o expresar resistencia intrínseca a una amplia variedad de antimicrobianos en uso clínico. Exhibe alto nivel de resistencia a la mayoría de las cefalosporinas, a todas las penicilinas semisintéticas, clindamicina, aminoglucósidos y a cotrimoxazol ${ }^{10}$. Adicionalmente, es tolerante a los antimicrobianos que inhiben la síntesis de la pared celular como son los $\beta$-lactámicos y glicopéptidos ${ }^{11}$. Por otro lado, cepas resistentes a vancomicina, ampicilina o aminoglucósidos, son aisladas cada vez con mayor frecuencia, y también se ha descrito resistencia a los nuevos fármacos con actividad anti-VRE como daptomicina, tigeciclina y linezolid ${ }^{10}$.

Históricamente, el manejo de las infecciones enterocócicas ha sido difícil. En la década del '50, se describió el fracaso terapéutico de la monoterapia con penicilina o estreptomicina para el tratamiento de la endocarditis ${ }^{12,13}$. 
Esto impulsó diferentes investigaciones, principalmente con modelos in vitro, con las cuales se definieron nuevas terapias con efecto sinérgico bactericida ${ }^{14}$. Por esta razón, durante décadas las guías para el tratamiento de la endocarditis infecciosa han recomendado la terapia combinada como primera línea ${ }^{15}$.

El propósito de esta revisión es analizar y resumir los resultados obtenidos de diferentes modelos experimentales de combinaciones anti-enterocócicas y de los estudios clínicos disponibles en PubMed/Medline, a fin de evaluar mejor la evidencia que soporta la utilización de estas combinaciones.

\section{Métodos}

\section{Fuente de datos}

Se realizó la búsqueda de artículos indexados en la base de datos PubMed/Medline en inglés, entre 1940 y 2017. Los términos utilizados fueron: ("Enterococcus/drug effects"[Mesh] AND "Drug Therapy, Combination"[Mesh]) AND ("Comparative Study"[All Fields] OR "pharmacodynamics "[All Fields] OR "pharmacokinetics "[All]). Si durante la revisión y análisis de los artículos seleccionados se encontraba una referencia a un trabajo adicional de interés que no había sido detectado con la estrategia de búsqueda, se decidió incluirlo para fortalecer la revisión.

\section{Criterios de exclusión}

Se excluyeron los artículos que no involucraron infecciones enterocócicas sistémicas, utilizaron modelos no mamíferos, emplearon antimicrobianos en monoterapia o sin aplicabilidad clínica, estudiaron sustancias diferentes a antimicrobianos, o artículos sin acceso a texto completo.

\section{Análisis de datos}

La siguiente información fue obtenida y tabulada a partir de los artículos revisados: (i) título del artículo, (ii) combinación antimicrobiana estudiada, (iii) cepas de Enterococcus, (iv) tipo de estudio: in vitro, in vivo o ensayo clínico, (v) infecciones enterocócicas endocárdicas o sistémicas no endocárdicas, (vi) efecto de la combinación, (vii) año de publicación, y (viii) revista. Los resultados obtenidos se presentan en forma descriptiva, incluyendo promedios y porcentaje cuando aplique. Ninguna prueba estadística fue aplicada, debido a la heterogeneidad de la información.

\section{Resultados}

Se encontraron 75 artículos, de los cuales se excluyeron 24 , porque en 12 de estos las combinaciones estudiadas no eran de antimicrobianos en uso clínico, en cuatro probaron únicamente monoterapia, tres de ellos emplearon modelos en invertebrados, dos incluían infecciones no sistémicas y en tres artículos la versión completa fue inaccesible.

\section{Pruebas in vitro para definir sinergismo}

Históricamente, las recomendaciones para el tratamiento combinado de infecciones enterocócicas se han basado en resultados de estudios in vitro. Inicialmente se probaron las combinaciones entre penicilina o aminopenicilina más estreptomicina o gentamicina, con un efecto bactericida in vitro, el cual fue corroborado por datos clínicos ${ }^{16,17}$. Estos hallazgos llevaron a los médicos a emplear la combinación ampicilina-gentamicina (AG) como el estándar de atención para la $\mathrm{EI}^{14,18}$.

En 1983, 30 años después de instaurarse el tratamiento combinado, Mederski y cols., reportaron las primeras cepas HLAR (en inglés, high-level aminoglycoside resistance $)^{19}$, lo que obligó a buscar nuevas opciones terapéuticas. En 1995 la combinación entre dos $\beta$-lactámicos surgió como una nueva alternativa. Mainardi y cols., describieron el efecto sinérgico in vitro de amoxicilina más cefotaxima contra $E$. faecalis $^{20}$. Posteriores estudios in vitro, in vivo y algunos estudios clínicos observacionales, demostraron el efecto sinérgico de la combinación ampicilina-ceftriaxona $(\mathrm{AC})^{21-23}$.

Aunque se han descrito varios métodos para medir el sinergismo in vitro, la técnica del tablero de ajedrez (en inglés, checkerboard) y las curvas de letalidad (en inglés time-kill curves) son las dos más utilizadas. El checkerboard (CB) mide el potencial de una combinación de antibacterianos para inhibir el crecimiento visible de un microorganismo. Los resultados se expresan en términos del índice de concentración inhibitoria fraccional (FIC index):

FIC index $=\mathrm{FIC}_{\mathrm{A}}+\mathrm{FIC}_{\mathrm{B}}=\frac{\mathrm{A}}{\mathrm{CIM}_{\mathrm{A}}}+\frac{\mathrm{B}}{\mathrm{CIM}_{\mathrm{B}}}(\mathrm{ec} .1)$

Donde A y B son las concentraciones inhibitorias mínimas (CIMs) de cada antibacteriano en combinación y $\mathrm{CIM}_{\mathrm{A}}$ y $\mathrm{CIM}_{\mathrm{B}}$ son las concentraciones inhibitorias mínimas de cada fármaco. Cuando el índice FIC es $\leq 0,5$, la combinación es sinérgica, entre 1 y 4 es indiferente y $>4$ es antagónica ${ }^{24,25}$.

Las time-kill curves (TKC) son un método dinámico que evalúa la actividad bactericida de la combinación de antibacterianos a lo largo del tiempo ${ }^{26}$. Si la combinación genera una reducción del recuento bacteriano mayor o igual a $2 \log _{10} u f c / m L$ que el fármaco individual más activo, se considera que hay sinergismo ${ }^{27}$.

Aunque estos métodos se utilizan frecuentemente, algunos autores han cuestionado la reproducibilidad de los datos y su potencial extrapolación ${ }^{28}$. Bayer y Morrison 
emplearon ambos métodos para determinar el sinergismo de vancomicina más rifampicina en aislados clínicos de Staphylococcus aureus sensibles (SASM) y resistentes a meticilina (SARM). Por TKC, la combinación fue indiferente con $67 \%$ de las cepas, sinérgica contra $19 \%$ y antagónica contra 14\%; mientras en el CB se observó antagonismo con $100 \%$ de los aislados. Los autores concluyeron que los resultados de las interacciones eran dispares y sugieren establecer comparaciones con modelos animales y ensayos clínicos controlados ${ }^{29}$. Con resultados similares, Rian y cols. ${ }^{30}$, estudiaron la variación de estos métodos in vitro contra Enterococcus, al combinar penicilina con seis aminoglucósidos. Por TKC se encontró sinergismo entre gentamicina y penicilina, mientras que con $\mathrm{CB}$ los resultados fueron muy variables.

La disparidad de resultados entre estos métodos puede explicarse por la ausencia de una metodología universalmente validada. La mayoría de los protocolos son adaptados o modificados a necesidad por los investigadores, lo cual dificulta la comparación de los resultados. Los cambios en los rangos de concentración de los fármacos son las modificaciones más comunes. Por ejemplo, algunos autores que emplean TKC utilizan concentraciones sub-CIM ${ }^{31}$, mientras que otros investigadores sostienen que las concentraciones a utilizar se deben basar en las concentraciones séricas de los fármacos, siendo este tipo de diseño el más usado ${ }^{32-34}$.

Los tiempos de incubación no están estandarizados en los estudios con TKC. El estudio de Whitman y cols., empleó un punto de corte de $18 \mathrm{~h}$, con el que se pudo observar el sinergismo entre ciprofloxacina más rifampicina o gentamicina versus $E$. faecium resistente a vancomicina $^{31}$. Por otro lado, Westh y cols., encontraron sinergismo entre tobramicina y tres $\beta$-lactámicos versus E. faecalis con solo cinco horas de incubación ${ }^{35}$.

Otras modificaciones no menos importantes incluyen la selección del medio de cultivo. La mayoría emplea caldo Mueller Hinton ajustado con cationes o de infusión cerebro corazón (BHI). Adicionalmente, algunos protocolos emplean medios suplementados con suero humano, de conejo o de rata, los que pueden cambiar el perfil sinérgico de una combinación ${ }^{36}$. Un estudio de ramoplanina más ampicilina versus $E$. faecium multi-resistente no evidenció sinergismo en las muestras con caldo Mueller Hinton ajustado con cationes, pero cuando se suplementó con $50 \%$ de suero humano, la combinación fue sinérgica ${ }^{37}$.

La Tabla 1 resume los estudios de sinergismo in vitro. La TKC fue la técnica más utilizada ${ }^{38,39}$. Las combinaciones más frecuentes fueron entre aminoglucósidos y aminopenicilinas $^{35,40,41}$, seguida por las combinaciones de dos $\beta$-lactámicos contra cepas de Enterococcus con $\mathrm{HLAR}^{21}$, y finalmente entre glicopéptidos y aminoglucósidos contra cepas VRE $\mathrm{VR}^{32,42,43}$.

\section{Modelos experimentales de infección en animales}

En 1899 MacCallum y Hastings, realizaron el primer modelo animal de infección por Enterococcus ${ }^{44}$. Desde entonces, estos modelos que utilizan principalmente conejos, ratas y ratones, han contribuido para superar la visión limitada y artificial de los modelos in vitro, y han permitido estudiar la patogénesis enterocócica en el contexto de la respuesta inmune, evaluar los efectos bacterianos en los diferentes órganos y comprender el entorno dinámico en el que interactúan el hospedero, el patógeno y los agentes antimicrobianos ${ }^{45,46}$

Existen varios modelos experimentales de infección sistémica, que incluyen los múridos de infección del tracto urinario (ITU), peritonitis en ratas y ratones, pero quizá, el modelo más utilizado es el de endocarditis en ratas y conejos ${ }^{27,45,47,48}$. Este último se ha empleado principalmente en estudios de patogenicidad y respuesta al tratamiento antimicrobiano ${ }^{49,50}$.

Por ejemplo, Gavaldà y cols., evaluaron la actividad del esquema AC contra $E$. faecalis con HLAR en un modelo de endocarditis en conejos. Las vegetaciones cardiacas de los animales tratados con AC, tuvieron conteos bacterianos más bajos que los tratados con ampicilina sola ${ }^{21}$. En otro estudio de EI en conejos, compararon el tratamiento de AC versus AG contra E. faecalis con o sin HLAR. Concluyeron que las dos combinaciones eran comparables con relación a la reducción de los recuentos bacterianos, y proponen AC como una alternativa al esquema AG en pacientes con insuficiencia renal ${ }^{51}$. Otros resultados del efecto de combinaciones anti-enterocócicas en el modelo de EI, se resumen en la Tabla 2.

\section{Farmacodinamia antimicrobiana}

Uno de los conceptos modernos más importantes de la farmacodinamia antimicrobiana es la relación de los parámetros farmacocinéticos $(\mathrm{PK})$ con los farmacodinámicos (PD) y la estimación de los índices PK/PD, con los cuales se puede predecir la eficacia bactericida in $v_{i v o}{ }^{52,53}$. La información obtenida de estas integraciones a partir de modelos animales de infección, ha facilitado el diseño de regímenes de dosificación óptimos, con potencial para reducir la toxicidad y el desarrollo de resistencia ${ }^{52}$. Algunos estudios de pacientes en tratamiento antimicrobiano han aportado información de los índices $\mathrm{PK} / \mathrm{PD}$ en humanos, con lo cual se ha confirmado que las magnitudes de los índices obtenidos en modelos animales y los encontrados en infecciones humanas son muy similares ${ }^{54,55}$.

Si bien el modelo de endocarditis ha contribuido al conocimiento de las interacciones farmacodinámicas de 


\begin{tabular}{|c|c|c|c|c|}
\hline Autor (año) & Técnica & Especie/cepa de enterococo & Combinación & Resultado \\
\hline Oill et al. (1981) & $C B$ & E. faecalis & $\mathrm{STR}+(\mathrm{AMP} \circ \mathrm{RIF})$ & Sinergismo \\
\hline Fass et al. (1984) & TKC & E. faecalis & $\mathrm{AMP}+\mathrm{GNT}$ & Sinergismo \\
\hline Peterson et al. (1987) & $C B$ & E. faecalis & $A Z L+(C I P \circ A M K)$ & Indiferente \\
\hline Sapico (1988) & TKC & E. faecalis & $\mathrm{DAP}+\mathrm{GNT}$ & Indiferente \\
\hline Duez et al. (1989) & $\mathrm{CB}$ & E. faecalis & $\mathrm{DAP}+\mathrm{AMP}$ & Sinergismo \\
\hline Westh et al. (1991) & TKC & E. faecalis & $\mathrm{TOB}+($ AMP o AMX o PEN-G) & Sinergismo \\
\hline Caron et al. (1991) & TKC & E. faecium & $\begin{array}{l}\text { LoD-PEN + (VAN o GNT) } \\
\text { HiD-PEN + (VAN o GNT) }\end{array}$ & $\begin{array}{l}\text { Sinergismo LoD-PEN + VAN } \\
\text { Sinergismo HiD-PEN + GNT }\end{array}$ \\
\hline Ramos et al. (1992) & TKC & E. faecalis PEN, GNT (R) & $\mathrm{AMP}+\mathrm{GNT}$ & Indiferente \\
\hline Bauernfeind et al.(1992) & TKC & E. faecalis AMP (R) & TEC + (CIP o NTM) & Sinergismo \\
\hline Caron et al. (1992) & TKC & E. faecium VRE & GNT + (DAP o TEC) & Sinergismo TEC + GNT \\
\hline Whitman et al. (1993) & TKC & E. faecium VRE & $\mathrm{CIP}+(\mathrm{RIF} \circ \mathrm{GNT})$ & Sinergismo \\
\hline Hayden et al. (1994) & TKC & E. faecium VRE & VAN + AMP & Indiferente \\
\hline Gavaldà et al. (1996) & TKC & E. faecalis & $\mathrm{AMP}+\mathrm{GNT}$ & Sinergismo \\
\hline Mercier et al. (1997) & TKC & E. faecium VRE & $\mathrm{ORV}+\mathrm{GNT}$ & Sinergismo \\
\hline Zinner et al. (1998) & TKC & E. faecium VRE & SAM + TVX & Indiferente \\
\hline Join-Lambert et al. (1998) & TKC & E. faecalis, HLAR- E. faecalis & $\mathrm{AMX}+\mathrm{CTX}$ & Sinergismo \\
\hline Baltch et al. (1998) & TKC & E. faecium VRE & ORV + AMP & Sinergismo \\
\hline Gavaldà et al. (1999) & TKC & E. faecalis HLAR & $\mathrm{AMP}+\mathrm{CRO}$ & Sinergismo \\
\hline Messick et al (1999) & TKC & E. faecium MDR & $\mathrm{CHL}+\mathrm{Q} / \mathrm{D}+($ VAN o AMP o GNT $)$ & Indiferente \\
\hline Mercier et al. (2002) & TKC & E. faecium VRE & $\mathrm{TG}+($ VAN o GNT o RIF $)$ & Indiferente \\
\hline Aslangul et al. (2005) & TKC & E. faecalis & GNT + AMX & Sinergismo \\
\hline Pontikis et al. (2013) & TKC & E. faecium $L Z(R)$ & $\mathrm{TG}+\mathrm{GNT}$ & Indiferente \\
\hline \multicolumn{5}{|c|}{$\begin{array}{l}\text { TKC: time-kill curves; CB: checkerboard; TG: tigeciclina; GNT: gentamicina; VAN: vancomicina; RIF: rifampicina; CHL: cloranfenicol; Q/D: quinupristina/dalfopristina; CRO: cef- } \\
\text { triaxona; ORV: oritavancina; CTX: cefotaxima; SAM: ampicilina/sulbactam; TVX: trovafloxacina; AZL: azlocilina; AMK: amikacina; CIP: ciprofloxacina; DAP: daptomicina; } \\
\text { NTM: netilmicina; TEC: teicoplanina; LOD-PEN: penicilina en bajas concentraciones; HID-PEN: penicilina en altas concentraciones; TOB: tobramicina; STR: estreptomicina; } \\
\text { VRE: vancomycin-resistant Enterococcus; HLAR: high-level aminoglycoside resistance; R: resistente; MDR: multidrug resistant. }\end{array}$} \\
\hline
\end{tabular}

combinaciones antibacterianas, también presenta grandes limitaciones metodológicas, que dificultan hacer una correcta predicción de la eficacia clínica del tratamiento ${ }^{56}$. Por ejemplo, no se cuantifica el tamaño del inóculo al momento del inicio de la infección. Este valor es de suma importancia para determinar el crecimiento bacteriano, el efecto del inóculo y el efecto post-antibiótico para algunos fármacos. Adicionalmente, con este modelo no se obtienen curvas farmacodinámicas dosis-respuesta, y por lo tanto, no se pueden estimar los índices PK/PD necesarios para determinar la eficacia bactericida in vivo. Por otro lado, las tasas bajas de multiplicación en las vegetaciones del microorganismo $\left(\sim 1 \log _{10} \mathrm{ufc} / \mathrm{g}\right)$, puede alterar la PD de algunos antibacterianos ${ }^{57,58}$; y finalmente estos modelos presentan varianzas grandes que reducen la potencia estadística ${ }^{56}$.

En un estudio, reciente optimizamos el modelo de ratón neutropénico para Enterococcus. Se ajustaron las condiciones in vitro de preparación del inóculo, lo cual mejoró el crecimiento bacteriano in vivo $>2 \log _{10}$ ufc/g en $24 \mathrm{~h}$ y se lograron estimaciones significativas de los parámetros PK/PD para vancomicina (VAN), ampicilina-sulbactam (SAM) y piperacillina-tazobactam (TZP), constituyéndose como el primer estudio in vivo en definir la magnitud del índice $f$ AUC/MIC de VAN contra E. faecium ${ }^{59}$. 


\begin{tabular}{|c|c|c|c|c|}
\hline $\begin{array}{l}\text { Referencia } \\
\text { (año) }\end{array}$ & Especie/cepa de enterococo & Combinación & Modelo & $\begin{array}{l}\text { Reducción de los recuentos bacterianos } \\
(\log 10 \mathrm{ufc} / \mathrm{g})\end{array}$ \\
\hline $\begin{array}{l}\text { Whitman et al. } \\
\text { (1993) }\end{array}$ & E. faecium VRE & $\mathrm{CIP}+($ RIF o GNT o RIF + GNT) & $\begin{array}{l}\text { El en rata. Tratamiento } \\
\text { por } 5 \text { días }\end{array}$ & $\begin{array}{l}\operatorname{GNT}(0), \operatorname{CIP}(1,2), \operatorname{RIF}(5,4), \operatorname{CIP}+\operatorname{GNT}(3,7) \\
\operatorname{CIP}+\operatorname{RIF}(5,9), \mathrm{CI}+\mathrm{GNT}+\operatorname{RIF}(6,3)\end{array}$ \\
\hline $\begin{array}{l}\text { Gavaldà et al. } \\
\text { (1996) }\end{array}$ & E. faecalis & $\mathrm{AMP}+\mathrm{GNT}$ & $\begin{array}{l}\text { El en conejo. Tratamiento } \\
\text { por } 5 \text { días }\end{array}$ & $\operatorname{AMP}(3,53), \quad A M P+\operatorname{GNT}(5,28)$ \\
\hline $\begin{array}{l}\text { Join-Lambert et al. } \\
\text { (1998) }\end{array}$ & E. faecalis y E. faecalis HLAR & $\mathrm{AMX}+\mathrm{CTX}$ & $\begin{array}{l}\text { El en conejo. Tratamiento } \\
\text { por } 5 \text { días }\end{array}$ & $\begin{array}{l}\text { E. faecalis } \operatorname{CTX}(0,2), \operatorname{AMX}(2,2), \operatorname{AMX}+\operatorname{CTX}(2) \\
\text { E. faecalis } \operatorname{HLRA} \operatorname{AMX}(3,6), \operatorname{AMX}+\operatorname{CTX}(2,8)\end{array}$ \\
\hline $\begin{array}{l}\text { Gavaldà et al. } \\
\text { (1999) }\end{array}$ & E. faecalis HLAR & $\mathrm{AMP}+\mathrm{CRO}$ & $\begin{array}{l}\text { El en conejo. Tratamiento } \\
\text { por } 3 \text { días }\end{array}$ & $\operatorname{AMP}(4,5), \operatorname{AMP}+\operatorname{CRO}(7,4)$ \\
\hline
\end{tabular}

GNT: gentamicina; VAN: vancomicina; RIF: rifampicina; CRO: ceftriaxona; ARK: arbekacina; CTX: cefotaxima; CIP: ciprofloxacina; LoD-PEN: penicilina a bajas concentraciones; HID-PEN: penicilina a altas concentraciones; VRE: vancomycin-resistant Enterococcus; HLAR: high-level aminoglycoside resistance; El: endocarditis infecciosa.

\section{¿Son comparables los resultados de los modelos de sinergismo in vitro e in vivo?}

Algunos de los datos reportados de estudios de combinaciones in vitro son discordantes con los hallazgos obtenidos en los modelos animales de infección. Por ejemplo, en las TKC y otras técnicas in vitro el inóculo bacteriano es expuesto a una concentración fija de antibacterianos durante un período de incubación ${ }^{60}$. Por otro lado, en los modelos in vivo, las concentraciones de los antibacterianos están cambiando continuamente en suero y en el tejido infectado. De esta manera, las bacterias en el sitio de infección están expuestas a concentraciones oscilantes de los fármacos. Finalmente, la definición de sinergismo en los modelos in vivo no es clara y no se ha establecido cuál es el punto de corte que permita definir si una combinación antibacteriana es sinérgica o no.

La Tabla 3 compara los resultados obtenidos de modelos in vivo e in vitro en combinaciones anti-enterocócicas. Whitman y cols. ${ }^{31}$, estudiaron el efecto de la combinación entre ciprofloxacina (CIP) más rifampicina (RIF) o gentamicina (GNT) contra E. faecium multi-resistente. Aunque por TKC las dos combinaciones fueron sinérgicas bactericidas (reducción de los recuentos bacterianos $\geq 8 \log _{10}$ ufc/mL), con el modelo de EI en rata, la disminución en los recuentos bacterianos de CIP + RIF y CIP + GNT en comparación con CIP fueron tan solo de 0,8 y 2,5 $\log _{10} \mathrm{ufc} / \mathrm{g}$, respectivamente. En otro estudio, Gavaldà y cols. ${ }^{51}$, evaluaron la eficacia in vivo e in vitro de los esquemas AC o AG versus E. faecalis. Por TKC todas las combinaciones fueron sinérgicas, mientras que, con el modelo de endocarditis, la disminución en los recuentos bacterianos de AG, AC y AGC (combinación triple ampicilina, gentamicina y ceftriaxona) en comparación con ampicilina en monoterapia fueron de 1,2-1,7 y 1,8 $\log _{10} \mathrm{ufc} / \mathrm{g}$, respectivamente. Sin embargo, los autores concluyen que hay evidencia de sinergismo in vitro e in vivo en todas las combinaciones probadas.

\section{Estudios clínicos}

Los estudios clínicos de infecciones por Enterococcus son limitados y se han restringido a la evaluación del esquema $\mathrm{AC}$ en EI contra cepas de E. faecalis HLAR no-HLAR (Tabla 4). En el año 2007, Gavaldà y cols. ${ }^{22}$, evaluaron la efectividad y seguridad del esquema AC en 21 pacientes con EI por E. faecalis HLAR y en 22 pacientes no-HLAR, en un ensayo clínico abierto multicéntrico. Concluyeron que el esquema $\mathrm{AC}$ extiende las opciones terapéuticas para el tratamiento de la EI por E. faecalis HLAR y No-HLAR sensibles a penicilina. Además, proponen este esquema como una alternativa de tratamiento para pacientes con riesgo de nefrotoxicidad infectados con cepas no-HLAR.

En 2013, Fernández-Hidalgo y cols. ${ }^{61}$, realizaron un es- 
Tabla 3. Comparación modelos in vivo e in vitro de combinaciones anti-enterocócicas

\begin{tabular}{|c|c|c|c|}
\hline \multirow[t]{2}{*}{$\begin{array}{l}\text { Referencia } \\
\text { (año) }\end{array}$} & \multirow[t]{2}{*}{ Especie/cepa de enterococo } & \multicolumn{2}{|c|}{$\begin{array}{l}\text { Reducción de los recuentos bacterianos (log10 ufc) de la combinación en comparación con el } \\
\text { fármaco único más activo }\end{array}$} \\
\hline & & Modelo in vivo de El & TKC \\
\hline Whitman et al. (1993) & E. faecium VRE & $\begin{array}{l}\text { CIP }+ \text { RIF 0,8 } \log 10 \mathrm{ufc} / \mathrm{g} \\
\mathrm{CIP}+\mathrm{GNT} 2,5 \log 10 \mathrm{ufc} / \mathrm{g} \\
\mathrm{CIP}+\mathrm{GNT}+\mathrm{RIF} 1,2 \log 10 \mathrm{ufc} / \mathrm{g}\end{array}$ & $\begin{array}{l}\mathrm{CIP}+\mathrm{RIF} \geq 8 \log 10 \mathrm{ufc} / \mathrm{mL} \\
\mathrm{CIP}+\mathrm{GNT} \geq 8 \log 10 \mathrm{ufc} / \mathrm{mL} \\
\mathrm{CIP}+\mathrm{GNT}+\mathrm{RIF} \geq 8 \log 10 \mathrm{ufc} / \mathrm{mL}\end{array}$ \\
\hline Gavaldà et al. (1996) & E. faecalis & AMP + GNT 1,75 $\log 10 u f c / g$ & AMP + GNT $3 \log 10 \mathrm{ufc} / \mathrm{mL}$ \\
\hline Join-Lambert et al. (1998) & E. faecalis y $E$. faecalis HLAR & $\begin{array}{l}\text { E. faecalis AMX + CTX 0,2 log } 10 \mathrm{CFU} / \mathrm{g} \\
\text { E. faecalis HLAR AMX + CTX 0,8 } \log 10 \mathrm{ufc/g}\end{array}$ & $\begin{array}{l}\text { E. faecalis AMX + CTX } 6 \log 10 \mathrm{ufc} / \mathrm{mL} \\
\text { E. faecalis HLAR AMX }+ \text { CTX } 6,5 \log 10 \mathrm{ufc} / \mathrm{mL}\end{array}$ \\
\hline Gavaldà et al. (1999) & E. faecalis HLAR & $\mathrm{AMP}+\mathrm{CRO} 2,6 \log 10 \mathrm{ufc} / \mathrm{g}$ & $\mathrm{AMP}+\mathrm{CRO} 3,4 \log 10 \mathrm{ufc} / \mathrm{mL}$ \\
\hline Gavaldà et al. (2003) & E. faecalis & $\begin{array}{l}\text { AMP }+ \text { GNT 1,2 } \log 10 u f c / g \\
A M P+C R O 1,7 \log 10 u f c / g\end{array}$ & $\begin{array}{l}\mathrm{AMP}+\mathrm{GNT} \geq 3 \log 10 \mathrm{ufc} / \mathrm{mL} \\
\mathrm{AMP}+\mathrm{CRO} \geq 3 \log 10 \mathrm{ufc} / \mathrm{mL}\end{array}$ \\
\hline
\end{tabular}

GNT: gentamicina; RIF: rifampicina; CRO: ceftriaxona; CTX: cefotaxima; CIP: ciprofloxacina; AMP: ampicilina; AMX: amoxicilina; VRE: vancomycin resistant Enterococcus; HLAR: high-level aminoglycoside resistance; El: endocarditis infecciosa; ufc, unidades formadoras de colonia; TKC: time kill curves.

Tabla 4. Ensayos clínicos para el tratamiento combinado de endocarditis infecciosa por Enterococcus

\begin{tabular}{|c|c|c|c|c|}
\hline Autor (año) & Esquema & Diseño & Sujetos & Resultados \\
\hline $\begin{array}{l}\text { Gavaldà et al. } \\
\text { (2007) }\end{array}$ & $\begin{array}{l}\text { AMP } 2 \mathrm{~g} \text { q4h más CRO } \\
2 \mathrm{~g} \mathrm{q} 12 \mathrm{~h}\end{array}$ & $\begin{array}{l}\text { Estudio observacional, abierto y } \\
\text { multicéntrico }\end{array}$ & $\begin{array}{l}43 \text { pacientes con El por } \\
\text { E. faecalis de } 13 \text { centros de } \\
\text { salud de España ( } 21 \text { HLAR } \\
\text { y } 22 \text { no-HLAR) }\end{array}$ & $\begin{array}{l}\text { Cura clínica de } 67,4 \% \\
28,6 \% \text { del grupo de pacientes HLAR y } 36,4 \% \text { del } \\
\text { grupo no-HLAR, murieron durante el tratamiento } \\
(p>0,05) \\
95,3 \% \text { de los pacientes no reportaron eventos } \\
\text { adversos }\end{array}$ \\
\hline $\begin{array}{l}\text { Fernández et al. } \\
\text { (2013) }\end{array}$ & $\begin{array}{l}\text { AMP } 2 \mathrm{~g} \text { q4h más CRO } \\
2 \mathrm{~g} \mathrm{q} 12 \mathrm{~h} \quad(\mathrm{n}=159) \\
\text { AMP } 2 \mathrm{~g} \text { q4h más GNT } \\
3 \mathrm{mg} / \mathrm{kg} / \text { día } \quad(\mathrm{n}=87)\end{array}$ & $\begin{array}{l}\text { Estudio observacional, abierto y } \\
\text { multicéntrico }\end{array}$ & $\begin{array}{l}246 \text { pacientes con El por } \\
\text { E. faecalis }\end{array}$ & $\begin{array}{l}\text { AC vs AG: Sin diferencias en la mortalidad durante } \\
\text { el tratamiento } \\
\text { AG: Aumento de eventos adversos que requirió la } \\
\text { suspensión del tratamiento ( } 25 \% \text { vs } 1 \%, p<0,001 \text { ) }\end{array}$ \\
\hline $\begin{array}{l}\text { Ceron et al. } \\
(2014)\end{array}$ & $\begin{array}{l}\text { DAP } \geq 6 \mathrm{mg} / \mathrm{kg} / \mathrm{día} \quad(\mathrm{n}=6) \\
\text { AMP } 2 \mathrm{~g} \text { q6h más CRO } \\
2 \mathrm{~g} \mathrm{q} 12 \mathrm{~h} \quad(\mathrm{n}=21) \\
\text { AMP } 2 \mathrm{~g} \text { q6h o VAN } 1 \mathrm{~g} \\
\text { q12h más GNT } 1 \mathrm{mg} / \mathrm{kg} \\
\text { q8h }(\mathrm{n}=5)\end{array}$ & $\begin{array}{l}\text { Estudio descriptivo retrospectivo } \\
\text { que compara la eficacia de DAP } \\
\text { vs AMP o AMP + CRO o VAN } \\
+ \text { GNT }\end{array}$ & $\begin{array}{l}32 \text { pacientes con El por } \\
\text { E. faecium }\end{array}$ & $\begin{array}{l}\text { Pacientes con DAP tuvieron bacteriemias más prolon- } \\
\text { gadas ( } 6 \text { días vs } 1 \text { día } p=0,01) \\
\text { Pacientes con DAP presentaron una necesidad ma- } \\
\text { yor de cambio de terapia debido a complicaciones } \\
(66,7 \% \text { vs } 0 \% \text {, p }<0,01) \text {. }\end{array}$ \\
\hline
\end{tabular}

tudio multicéntrico no aleatorizado, de cohortes, en el cual compararon la seguridad y eficacia de AC y AG en 246 pacientes con EI causada por E. faecalis ( 159 sujetos en el grupo AC y 87 en el grupo AG). Los autores concluyeron que las dos combinaciones eran igualmente efectivas. Sin embargo, los pacientes tratados con AG tuvieron tasas más altas de insuficiencia renal que requirieron la suspensión del tratamiento. Con los resultados de estos dos estudios clínicos, la Guía Americana de EI en Adultos de 2015 recomienda el tratamiento dual con $\beta$-lactámicos como una opción de tratamiento para las infecciones por HLAR y una alternativa razonable a las combinaciones con aminoglucósidos en infecciones por E. faecalis (Clase IIa; nivel de evidencia de recomendación B) ${ }^{15,62}$.

\section{Conclusiones}

Desde hace más de cinco décadas, la evidencia para el tratamiento combinado de infecciones graves entero- 
cócicas se ha obtenido principalmente de resultados de estudios in vitro, modelos de endocarditis en conejos y unos pocos estudios clínicos. Los hallazgos derivados de estas investigaciones dieron origen a los esquemas $\mathrm{AG}$ como tratamiento estándar para la EI, y la combinación dual con $\beta$-lactámicos (es decir, AC), para infecciones con cepas HLAR y para la disminución del riesgo de toxicidad renal por aminoglucósidos. Aunque con el uso de estos esquemas se han logrado mejores tasas de éxito terapéutico, no se ha esclarecido cuál es el tratamiento óptimo contra Enterococcus. Por lo tanto, existe una necesidad crítica de investigar, además de mejores esquemas de tratamiento, nuevos modelos experimentales in vivo que permitan predecir la eficacia clínica de las combinaciones.

La evidencia del efecto in vivo del tratamiento antienterocócico en monoterapia y en combinación, deriva principalmente del modelo de EI en conejos, el que puede ser diferente al obtenido en otras infecciones graves no endocárdicas. Por lo tanto, se requieren modelos animales validados que provean información fiable sobre la respuesta terapéutica en infecciones sistémicas graves y que los resultados sean extrapolables a humanos. El modelo de ratón neutropénico optimizado para Enterococcus obtuvo estimaciones significativas de los parámetros $\mathrm{PK} / \mathrm{PD}$ en monoterapia, constituyéndose como una herramienta promisoria para evaluar el efecto de las combinaciones anti-enterocócicas.

\section{Resumen}

Durante las últimas décadas, especies del género Enterococcus han emergido como importantes agentes etiológicos de bacteriemia, osteomielitis, endocarditis e infecciones de tejidos blandos. La combinación de antibacterianos ha sido la estrategia terapéutica más utilizada para dichas infecciones, buscando un potencial efecto sinérgico bactericida. Sin embargo, aparte de los modelos in vitro e in vivo, la utilidad clínica del tratamiento combinado genera controversia, especialmente en infecciones sistémicas no endocárdicas. Aunque las combinaciones entre $\beta$-lactámicos y aminoglucósidos o el tratamiento dual con $\beta$-lactámicos, han mejorado las tasas de curación de la endocarditis, aún no se ha esclarecido cuál es su tratamiento óptimo o si estas combinaciones también son útiles en otro tipo de infecciones graves sistémicas. El propósito de esta revisión es analizar y resumir los resultados obtenidos de diferentes modelos experimentales de combinaciones anti-enterocócicas y de los estudios clínicos disponibles en PubMed/Medline, a fin de evaluar mejor la evidencia que soporta la utilización de estas combinaciones. En conclusión, la información disponible es escasa, e indica la necesidad de mejores modelos in vivo y estudios clínicos que permitan comprobar la potencial actividad sinérgica de las combinaciones anti-enterocóciccas.

\section{Referencias bibliográficas}

1.- Centers for Disease Control and Prevention. Antibiotic resistance threats in the United States, 2013: Centres for Disease Control and Prevention, US Department of Health and Human Services. URL: https://www.cdc. gov/drugresistance/threat-report-2013/pdf/arthreats-2013-508.pdf.

2.- Arias C A, Murray B E. The rise of the Enterococcus: Beyond vancomycin resistance. Nat Rev Microbiol 2012;10 (4):266-78. doi:10.1038/nrmicro2761.

3.- Sievert D M, Ricks P, Edwards J R, Schneider M, Patel J, Srinivasan A, et al. Antimicrobialresistant pathogens associated with healthcareassociated infections summary of data reported to the National Healthcare Safety Network at the Centers for Disease Control and Prevention, 2009-2010. Infect Control Hosp Epidemiol. 2013; 34 (01): 1-14. doi:10.1086/668770.

4.- Zuluaga A F. Etiologic diagnosis of chronic osteomyelitis. Arch Intern Med 2006;166 (1): 95-100. doi:10.1001/archinte.166.1.95.

5.- Gilmore MS. MICROBIOLOGY: The thin line between gut commensal and pathogen. Science (80- ). 2003; 299 (5615):1999-2002. doi:10.1126/science.1083534.
6.- Miro J M, Pericas J M, del Río A. A New Era for treating Enterococcus faecalis endocarditis: ampicillin plus short-course gentamicin or ampicillin plus ceftriaxone: that is the question! Circulation 2013; 127 (17): 1763-6. doi:10.1161/CIRCULATIONAHA.113.002431.

7.- Dupre I. Incidence of virulence determinants in clinical Enterococcus faecium and Enterococcus faecalis isolates collected in Sardinia (Italy). J Med Microbiol 2003; 52 (6):491-8.

8.- Rosa R, Creti R, Venditti M, D’Amelio R, Arciola C R, Montanaro L, et al. Relationship between biofilm formation, the enterococcal surface protein (Esp) and gelatinase in clinical isolates of Enterococcus faecalis and Enterococcus faecium. FEMS Microbiol Lett. 2006; 256 (1): 145-50. -150. doi:10.1111/ j.1574-6968.2006.00112.x.

9.- Soares R O, Fedi A C, Reiter K C, Caierão J, D'Azevedo P A. Correlation between biofilm formation and gelE, esp, and agg genes in Enterococcus spp. clinical isolates. Virulence. 2014;5 (5): 634-7. doi:10.4161/viru.28998.

10.- Kristich C J, Rice L B, Arias C A. Enterococcal Infection-Treatment and Antibiotic Resistance.; 2014. In: Gilmore MS, Clewell DB, Ike Y, Shankar N, editors. Enterococci: From
Commensals to Leading Causes of Drug Resistant Infection [Internet]. Boston: Massachusetts Eye and Ear Infirmary. $2014 \mathrm{Feb}$ 6.PMID: 24649502

11.- Arias C A, Contreras G A, Murray B E. Management of multi-drug resistant enterococcal infections. Clin Microbiol Infect. 2010; 16 (6): 555-62. doi:10.1111/j.1198743X.2010.03214.x.

12.- Hunter T H. Use of streptomycin in the treatment of bacterial endocarditis. Am J Med 1947; 2 (5): 436-42. doi:10.1016/00029343(47)90088-0.

13.- Hunter $T H$. Bacterial endocarditis. Am Heart J 1951; 42 (3): 472-82. doi:10.1016/00028703(51)90144-5.

14.- Geraci J E, Martin W J. Antibiotic therapy of bacterial endocarditis. VI. Subacute enterococcal endocarditis; clinical, pathologic and therapeutic consideration of 33 cases. Circulation 1954; 10 (2): 173-94. doi: 10.1161/01.cir.10.2.173.

15.- Baddour L M, Wilson W R, Bayer A S, Fowler V G, Tleyjeh I M, Rybak M J, et al. Infective endocarditis in adults: diagnosis, antimicrobial therapy, and management of complications. Circulation 2015; 132 (15): 1435-86. doi: 10.1161/CIR.0000000000000296. 
16.- Jewetz E, Gunnison J B, Bruff J B, Coleman V R. Studies on antibiotic synergism and antagonism. Synergism among seven antibiotics against various bacteria in vitro. J Bacteriol 1952; 64 (1): 29-39. PMID:14946096.

17.- Robbins W C, Tompsett R. Treatment of enterococcal endocarditis and bacteremia. Am J Med 1951; 10(3): 278-99. -doi:10.1016/00029343(51)90273-2.

18.- Habib G, Lancellotti P, Antunes M J, Bongiorni M G, Casalta J-P, Del Zotti F, et al. 2015 ESC Guidelines for the management of infective endocarditis. Eur Heart J 2015; 36 (44): 3075 128. Available from: https://academic.oup.com/ eurheartj/article-lookup/doi/-3128. doi:10.1093/ eurheartj/ehv319.

19.- Mederski-Samoraj B D, Murray B E. Highlevel resistance to gentamicin in clinical isolates of enterococci. J Infect Dis 1983;147(4):751-7. doi: 10.1093/infdis/147.4.751.

20.- Mainardi J L, Gutmann L, Acar J F, Goldstein F W. Synergistic effect of amoxicillin and cefotaxime against Enterococcus faecalis. Antimicrob Agents Chemother 1995; 39 (9): 1984-7. doi: 10.1128/aac.39.9.1984.

21.- Gavaldà J, Torres C, Tenorio C, López P, Zaragoza M, Capdevila JA, et al. Efficacy of ampicillin plus ceftriaxone in treatment of experimental endocarditis due to Enterococcus faecalis strains highly resistant to aminoglycosides. Antimicrob Agents Chemother 1999; 43 (3): 639-46. doi:10.1128/ AAC.43.3.639.

22.- Gavaldà J, Len O, Miró JM, Muñoz P, Montejo M, Alarcón A, et al. Brief communication: treatment of Enterococcus faecalis endocarditis with ampicillin plus ceftriaxone. Ann Intern Med 2007; 146 (8): 574-9. doi: 10.7326/00034819-146-8-200704170-00008.

23.- Pericas J M, Cervera C, del Río A, Moreno A, García de la María C, Almela M, et al. Changes in the treatment of Enterococcus faecalis infective endocarditis in Spain in the last 15 years: from ampicillin plus gentamicin to ampicillin plus ceftriaxone. Clin Microbiol Infect 2014; 20 (12): O1075-83. doi: 10.1111/1469-0691.12756.

24.- Duez J M, Péchinot A, Siébor E, Cordin X, Kazmierczak A. Bactericidal activity of daptomycin and vancomycin alone or in combination with tobramycin, netilmicin or ampicillin against enterococcus. Pathol Biol (Paris) 1989; 37 (4): 263-8. PMID: 2543946.

25.- Yamashita K, Kawabe H, Mitsuhashi S. Synergistic activities of fortimicin A and beta-lactam antibiotics against Pseudomonas aeruginosa. Antimicrob Agents Chemother. 1981; 20 (1): 33-7. doi:10.1128/AAC.20.1.33.

26.- Pillai S K, Moellering R C E G. Antimicrobial Combinations. In: Lorian, V. Antibiotics in Laboratory Medicine. 5th ed. Philadelphia: The Lippincott Williams \& Wilkins Co.; 2005; 365-
$440 \mathrm{p}$.

27.- Leone S, Noviello S, Esposito S. Combination antibiotic therapy for the treatment of infective endocarditis due to enterococci. Infection. 2016; 44 (3): 273-81. doi: 10.1007/s15010-0150836-0.

28.- Norden C W, Wentzel H, Keleti E. Comparison of techniques for measurement of in vitro antibiotic synergism. J Infect Dis. 1979;140(4):629-33. doi:10.1093/ infdis/140.4.629.

29.- Bayer A S, Morrison J O. Disparity between timed-kill and checkerboard methods for determination of in vitro bactericidal interactions of vancomycin plus rifampin versus methicillin-susceptible and -resistant Staphylococcus aureus. Antimicrob Agents Chemother 1984; 26 (2): 220-3. doi:10.1128/ AAC.26.2.220.

30.- Ryan R W, Kwasnik I, Tilton R C. Methodological variation in antibiotic synergy tests against enterococci. J Clin Microbiol 1981; 13 (1):73-5. PMID: 6780605.

31.- Whitman M S, Pitsakis P G, Zausner A, Livornese L L, Osborne A J, Johnson C C, et al. Antibiotic treatment of experimental endocarditis due to vancomycin- and ampicillin-resistant Enterococcus faecium. Antimicrob Agents Chemother 1993; 37 (10): 2069-73. PMID: 8257125.

32.- Bauernfeind A. Bactericidal activity of antibiotics alone and in combination against Enterococcus faecalis in a pharmacodynamic model. Clin Infect Dis 1992; 15 (3): 481-5. doi: 10.1093/clind/15.3.481.

33.- Messick C R, Rodvold K A, Pendland S L. Modified time-kill assay against multidrugresistant Enterococcus faecium with novel antimicrobial combinations. J Antimicrob Chemother 1999; 4 (6): 831-4. doi: 10.1093/ jac/44.6.831.

34.- Zinner S H, Gilbert D, Dudley M N. Activity of trovafloxacin (with or without ampicillinsulbactam) against enterococci in an in vitro dynamic model of infection. Antimicrob Agents Chemother 1998; 42 (1): 72-7. doi: 10.1128/ AAC.42.1.72.

35.- Westh H, Frimodt-Møller N, Gutschik E. Bactericidal effect of penicillin, ampicillin, and amoxicillin alone and in combination with tobramycin against Enterococcus faecalis as determined by kill-kinetic studies. Infection. 1991; 19 (3): 170-3. PMID: 1909688.

36.- Baltch A L, Smith R P, Ritz W J, Bopp L H. Comparison of inhibitory and bactericidal activities and postantibiotic effects of LY333328 and ampicillin used singly and in combination against vancomycinresistant Enterococcus faecium. Antimicrob Agents Chemother 1998; 42 (10): 25648 PMID: 9756756

37.- Mobarakai N, Quale J M, Landman D.
Bactericidal activities of peptide antibiotics against multidrug-resistant Enterococcus faecium. Antimicrob Agents Chemother 1994; 38 (2): 385-7. PMID: 8192473.

38.- Hayden M K, Koenig G I, Trenholme G M. Bactericidal activities of antibiotics against vancomycin-resistant Enterococcus faecium blood isolates and synergistic activities of combinations. Antimicrob Agents Chemother. 1994; 38 (6): 1225-9. PMID: 8092818.

39.- Mercier R-C, Kennedy C, Meadows C. Antimicrobial activity of tigecycline (GAR936) against Enterococcus faecium and Staphylococcus aureus used alone and in combination. Pharmacotherapy. 2002; 22 (12): 1517-23. doi: 10.1592/phco.22.17.1517.34117.

40.- Fass R J, Wright C A. Comparative efficacies of mezlocillin and ampicillin alone or in combination with gentamicin in the treatment of Streptococcus faecalis endocarditis in rabbits. Antimicrob Agents Chemother 1984; 5 (4): 408-10. PMID: 6428306.

41.- Oill P A, Kalmanson G M, Guze L B. Rifampin, ampicillin, streptomycin, and their combinations in the treatment of enterococcal pyelonephritis in rats. Antimicrob Agents Chemother 1981; 20 (4): 491-2. PMID: 6805418.

42.- Caron F, Kitzis M D, Gutmann L, Cremieux A C, Maziere B, Vallois J M, et al. Daptomycin or teicoplanin in combination with gentamicin for treatment of experimental endocarditis due to a highly glycopeptide-resistant isolate of Enterococcus faecium. Antimicrob Agents Chemother 1992; 36 (12): 2611-6. doi: 10.1128/ AAC.36.12.2611.

43.- Mercier R C, Houlihan H H, Rybak M J. Pharmacodynamic evaluation of a new glycopeptide, LY333328, and in vitro activity against Staphylococcus aureus and Enterococcus faecium. Antimicrob Agents Chemother 1997; 41 (6): 1307-12. PMID: 9174189.

44.- Maccallum W G, Hastings T W. A case of acute endocarditis caused by Micrococcus zymogenes (nov. Spec.), with a description of the microorganism. J Exp Med. 1899; 4 (5-6): 521-34. doi: 10.1084/jem.4.5-6.521

45.- Garsin D A, Frank K L, Silanpää J, Ausubel F M, Hartke A, Shankar N, et al. Pathogenesis and models of enterococcal infection. In: Gilmore MS, Clewell DB, Ike Y, Shankar N, editors. Enterococci: From Commensals to Leading Causes of Drug Resistant Infection [Internet]. Boston: Massachusetts Eye and Ear Infirmary; 2014. 2014 Feb 7. 2014. PMID: 24649512.

46.- Renneberg J. Definitions of antibacterial interactions in animal infection models. J Antimicrob Chemother 1993; 31 (suppl D): 167-75. doi:10.1093/jac/31.suppl D.167.

47.- Ramos M C, Grayson M L, Eliopoulos G 
M, Bayer A S. Comparison of daptomycin, vancomycin, and ampicillin-gentamicin for treatment of experimental endocarditis caused by penicillin-resistant enterococci. Antimicrob Agents Chemother 1992; 36 (9): 1864-9. PMID: 1329632.

48.- Sapico F L, Ginunas V J, Canawati H N, Montgomerie J Z. LY146032, alone and in combination with gentamicin, for the treatment of enterococcal pyelonephritis in the rat model. Antimicrob Agents Chemother 1988; 32 (1): 81-3. doi:10.1128/AAC.32.1.81.

49.- Marangos M. Influence of gentamicin dosing interval on the efficacy of penicillincontaining regimens in experimental Enterococcus faecalis endocarditis. J Antimicrob Chemother 1997; 39 (4): 519- 22. doi:10.1093/jac/39.4.519.

50.- Pontikis K, Pefanis A, Tsaganos T, Tzepi I-M, Carrer D-P, Giamarellou H. Efficacy of tigecycline alone and in combination with gentamicin in the treatment of experimental endocarditis due to linezolid-resistant Enterococcus faecium. Antimicrob Agents Chemother 2013; 57 (7): 3392-4. doi:10.1128/ AAC.00646-13.

51.- Gavalda J. Efficacy of ampicillin combined with ceftriaxone and gentamicin in the treatment of experimental endocarditis due to Enterococcus faecalis with no high-level resistance to aminoglycosides. J Antimicrob Chemother 2003; 52 (3): 514-7. doi:10.1093/ $\mathrm{jac} / \mathrm{dkg} 360$.
52.- González J M, Rodríguez CA, Agudelo $\mathrm{M}$, Zuluaga A F, Vesga O. Antifungal pharmacodynamics: Latin America's perspective. Brazilian J Infect Dis 2017; 21(1): 79-87. doi:10.1016/j.bjid.2016.09.009.

53.- Craig W A. Pharmacokinetic/pharmacodynamic parameters: rationale for antibacterial dosing of mice and men. Clin Infect Dis 1998; 26 (1):112; https://doi.org/10.1086/516284.

54.- Zuluaga A F, OV. Aportes del modelo en ratón neutropénico de infección del muslo al conocimiento sobre la farmacología de antibióticos. Infectio 2005; 9. (2): 1-159. URL: http:// www.revistainfectio.org/index. php/infectio/article/download/202/226

55.- Ambrose P G, Bhavnani S M, Rubino C M, Louie A, Gumbo T, Forrest A, et al Antimicrobial resistance: Pharmacokineticspharmacodynamics of antimicrobial therapy: it's not just for mice anymore. Clin Infect Dis 2007; 44 (1): 79-86. doi:10.1086/510079.

56.- Zuluaga A F, Rodríguez C A, Agudelo M, Vesga $\mathrm{O}$. About the validation of animal models to study the pharmacodynamics of generic antimicrobials. Clin Infect Dis 2014; 59 (3): 459-61. doi:10.1093/cid/ ciu306.

57.- Caron F, Carbon C, Gutmann L. Triplecombination penicillin-vancomycin-gentamicin for experimental endocarditis caused by a moderately penicillin- and highly glycopeptide resistant isolate of Enterococcus faecium. J Infect Dis 1991; 164 (5): 888-93. doi:10.1093/ infdis/164.5.888.

58.- Kak V, Donabedian S M, Zervos M J, Kariyama R, Kumon H, Chow J W. Efficacy of ampicillin plus arbekacin in experimental rabbit endocarditis caused by an Enterococcus faecalis strain with high-level gentamicin resistance. Antimicrob Agents Chemother. 2000; 44 (9): 2545-6. doi:10.1128/ AAC.44.9.2545-2546.2000.

59.- Rodríguez C A, Agudelo M, González J M, Vesga O, Zuluaga A F. An optimized mouse thigh infection model for Enterococci and its impact on antimicrobial pharmacodynamics. Antimicrob Agents Chemother. 2015; 59 (1): 233-8. doi:10.1128/AAC.02352-13.

60.- Berenbaum M C. Correlations between methods for measurement of synergy. J Infect Dis 1980; 142 (3): 476-80. doi: 10.1093 infdis/142.3.476.

61.- Fernández-Hidalgo N, Almirante B, Gavaldà J, Gurgui M, Peña C, de Alarcón A, et al. Ampicillin plus ceftriaxone is as effective as ampicillin plus gentamicin for treating enterococcus faecalis infective endocarditis. Clin Infect Dis 2013; 56 (9): 1261-8. doi:10.1093/cid/cit052.

62.- Olaison L, Schadewitz K, Swedish Society of Infectious Diseases Quality Assurance Study Group for Endocarditis. Enterococcal endocarditis in Sweden, 1995-1999: can shorter therapy with aminoglycosides be used? Clin Infect Dis 2002; 34 (2): 159-66. doi:10.1086/338233. 